\title{
Electrospinning of Poly(ethylene-co-vinyl alcohol) Nanofibres Encapsulated with Ag Nanoparticles for Skin Wound Healing
}

\author{
Chao Xu, ${ }^{1,2}$ Feng Xu, ${ }^{1,3}$ Bin Wang, ${ }^{1,2}$ and TianJian Lu ${ }^{1}$ \\ ${ }^{1}$ Biomedical Engineering and Biomechanics Center, School of Aerospace, Xi'an Jiaotong University, Xi'an 710049, China \\ ${ }^{2}$ School of Engineering, University of Aberdeen, Aberdeen AB24 3UE, UK \\ ${ }^{3}$ Department of Medicine, HST-Center for Biomedical Engineering, Brigham and Women's Hospital, Harvard Medical School, \\ Boston, MA 02115, USA \\ Correspondence should be addressed to Bin Wang, bin.wang@abdn.ac.uk
}

Received 2 November 2010; Revised 14 December 2010; Accepted 17 December 2010

Academic Editor: Zuwei Ma

Copyright (๑) 2011 Chao Xu et al. This is an open access article distributed under the Creative Commons Attribution License, which permits unrestricted use, distribution, and reproduction in any medium, provided the original work is properly cited.

Skin wound healing is an urgent problem in clinics and military activities. Although significant advances have been made in its treatment, there are several challenges associated with traditional methods, for example, limited donor skin tissue for transplantation and inflammation during long-term healing time. To address these challenges, in this study we present a method to fabricate Poly(ethylene-co-vinyl alcohol) (EVOH) nanofibres encapsulated with Ag nanoparticle using electrospinning technique. The fibres were fabricated with controlled diameters $(59 \mathrm{~nm}-3 \mu \mathrm{m})$ by regulating three main parameters, that is, EVOH solution concentration, the electric voltage, and the distance between the injection needle tip (high-voltage point) and the fibre collector. $\mathrm{Ag}$ was added to the nanofibres to offer long-term anti-inflammation effect by slow release of Ag nanoparticles through gradual degradation of $\mathrm{EVOH}$ nanofibre. The method developed here could lead to new dressing materials for treatment of skin wounds.

\section{Introduction}

Skin wounds are a significant health problem, which negatively affect lives of millions of people worldwide inducing huge societal costs [1-3]. Every year in the USA, more than 1.25 million people have burns [4-6] and 6.5 million people have chronic skin ulcer caused by pressure, venous stasis, or diabetes mellitus [7]. Although skin wound healing has become an increasingly mature topic of study in clinics and academia $[8,9]$, there are only few breakthroughs in skin wound therapies, mainly due to limited capacities in mitigating body fluid loss and inflammation, particularly for damages of large skin areas.

Existing methods in skin wound treatment have shown steady progress, with increasing survival rate in large-scale burn injuries. There are broadly two types of treatment methods for skin wounds. The first is the medication method, in which coating medicines are applied onto wounded area and covered with bandages. Frequent dressing changes are required to clear body discharges and replace medicines. However, such frequent changes cause severe pains and increase the rate of inflammation. The second method is skin transplantation, which is more effective in both improving healing and decreasing infections. However, this method is associated with several limitations such as the shortage of donor skin tissues and high cost [10]. As such, skin dressing is still the most widely used treatment in clinical practice and will remain so in the foreseeable future. There is an unmet need for novel materials for better functionality, availability, and effectiveness in skin wound dressing.

In this study, we explored new nanofibres-based dressing materials with better clinical properties to address the problems associated with existing woven and nonwoven materials currently used in skin wound treatment. The advantages of nanofibres network over the traditional dressing materials come from its large surface/volume ratio [11]. This helps to promote permeation of water molecules through the dressing to regulate the moisture level at the wound surface, an important factor to promote cell growth and reduce inflammation. The large surface/volume ratio system also allows drugs, for example, antibacterial drugs or cell growth promoters, to be retained within the structure to control 


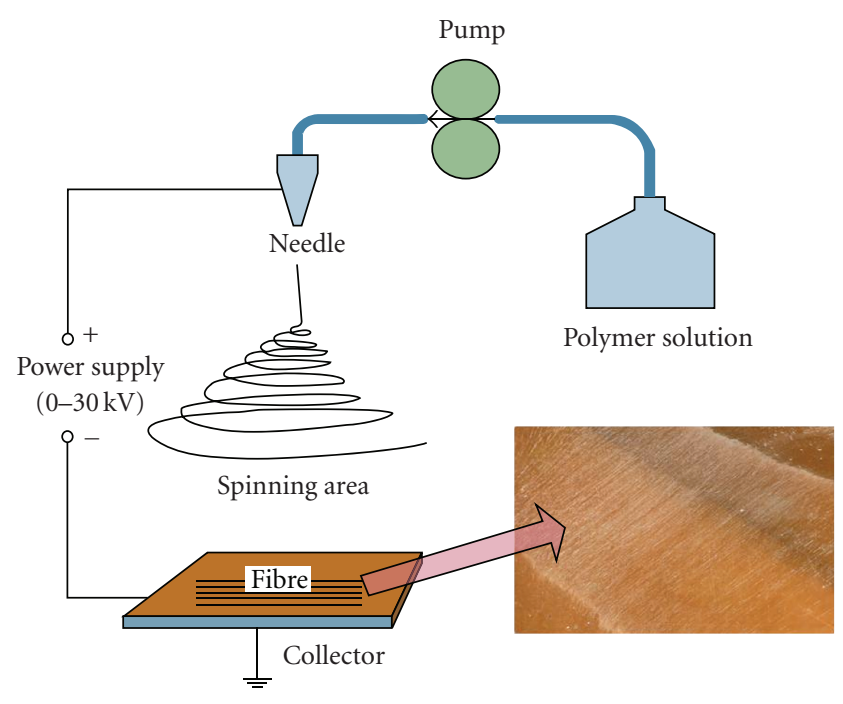

FIGURE 1: The electrospinning system. The electrospinning setup is composed of a syringe and blunt-end needle, a ground electrode as a collector, and a high-voltage supply with a low-current output (limited to a few $\mathrm{mA}$ ) to generate a static electric field.

infections and facilitate new growths. In addition, materials of proven biocompatibility and biodecomposability such as Poly-L-lactide acid (PLLA), Polyorthoester (POE), and Poly (L-lactide-co-glycolide) (PLGA) [12,13] can be selected for fabricating fibres encapsulated with drugs and controlling the release of the drugs. These advantages would lead to less frequency in dressing replacement and a better environment for wound healing.

Electrospinning phenomenon was firstly discovered in 1902 [14], where a high-voltage supply was used to stretch a polymer solution injected out of a fine needle. The phenomenon of Taylor Cone was discovered in 1969 by Sir Geoffrey Taylor. This phenomenon has been theoretically analysed, and it is shown that a conducting fluid can exist in equilibrium in the form of a cone under the action of electric field where a jet can be formed at the apex of the cone if a continuing supply of the liquid is provided [15]. In the past few years, significant advances have been achieved in this area, most noticeably in the manufacturing process of electrospun guidance nanofibres, the electrospinning of a bioceramic fibres process, and the technologies of coaxial electrospinning of producing nanotube fibres [16]. The fibre diameter has not been seen down to the size of a single nano, but diameters measured in tens of nanos are achievable (as in this study, we managed fibre diameter down to 50 nanos).

The fibre dries up or solidifies before being deposited onto the grounded collector. The setup used in this study is schematically shown in Figure 1. Note that due to the very low mass of the fibre, its spinning motion can be severely affected by even the smallest air flow in the "flight" space.

\section{Material and Electrospinning Methods}

2.1. Material. Poly(ethylene-co-vinyl alcohol) EVOH (Sigma-Aldrich, Batch number: 12822PE) was used to make nanofibres in this study for its good mechanical properties, the biocompatibility, and biodecomposability [17]. The Ag contents in the fibres were obtained from $99.9995 \%$ pure $\mathrm{AgNO}_{3}$ powders (Alfa Aesar, 7761-88-8) dissolved into the solution for electrospinning following standard protocol [18].

2.2. Preparation of EVOH Polymer Solution. Six different concentrations of EVOH solutions (2.5, 5.0, 7.5, 10.0, 12.5, and $15.0 \mathrm{wt} \%$ ) were prepared, which can be readily electrospun at the room temperature. To prepare an $\mathrm{EVOH}$ polymer solution, we diluted EVOH powders in solvent of propan-2$\mathrm{ol}$, and water. A variety of proportions of propan-2-ol and water were tried, and it is found that the combination of polymer EVOH, $80 \%$ propan-2-ol and $20 \%$ water heated at $80^{\circ} \mathrm{C}$ with a reflux setup for 2 to 3 hours produces the solvent for an optimal output of nanofibres. Although solutions of other solvent proportions can be electrospun into fibres, such as $\mathrm{EVOH}$ dissolved in 70\% 2-propanol/30\% water, they appear to be hindrance to the formation of $\mathrm{Ag}$ particles in fibres when $\mathrm{AgNO}_{3}$ was added, yielding in scattering $\mathrm{Ag}$ particle sizes and distributions.

2.3. Electrospinning Nanofibres. The system used to carry out the "spinning" is composed of a high power supply (Spellman CZE1000R, $0-30 \mathrm{kV}$,) with very low-current output ( $\mu \mathrm{A}$ ranges), a peristaltic pump (Masterflex, 77120-52) with a feeding capacity in the range of $1.0-15.0 \mathrm{~mL} / \mathrm{h}$, a container (such as a syringe) with a fine needle, which is charged by the power supply, and a metal collector (can be in various shapes) which is normally grounded. The charged tip and grounded collector form a static electric field between them to provide the driving force to spin the fibre.

2.4. Encapsulation of Ag Nanoparticles in the Fibre. The suppressive effect of Ag to inflammation is well understood and utilized in clinical practice. To encapsulate Ag nanoparticles into fibres, $\mathrm{AgNO}_{3}$ of various concentrations was first added into $7.5 \mathrm{wt} \% \mathrm{EVOH}$ solution which was then electrospun into nanofibres. $\mathrm{AgNO}_{3}$ in the fibres is deoxidized and forms into pure Ag nanoparticles when exposed to illumination. The speed of deoxidization can be accelerated by using UV lights. For fibres containing Ag, it was found that the collector is required to be changed from a plate shape to a stripe one without ground connection due to the increased conductivity of the fibre.

2.5. Characterization of the Fabricated Fibres. We fabricated nanofibres using different electrospinning parameters including the distance $(15-35 \mathrm{~cm})$ between the tip of the injecting needle and the collector, the voltage $(8-20 \mathrm{kV})$ applied to generate the electric field, and the density (2.5-15.0 wt\%) of the polymer solution. Scanning electron microscopy (JSM-6700 Cold FE SEM) was performed for fibre measurement. All fibre samples were mounted onto a copper stub and sputter coated with gold for energy spectrum analysis in SEM. Each sample was divided into 12 parts for diameter measurements. We used 12 uniform size 


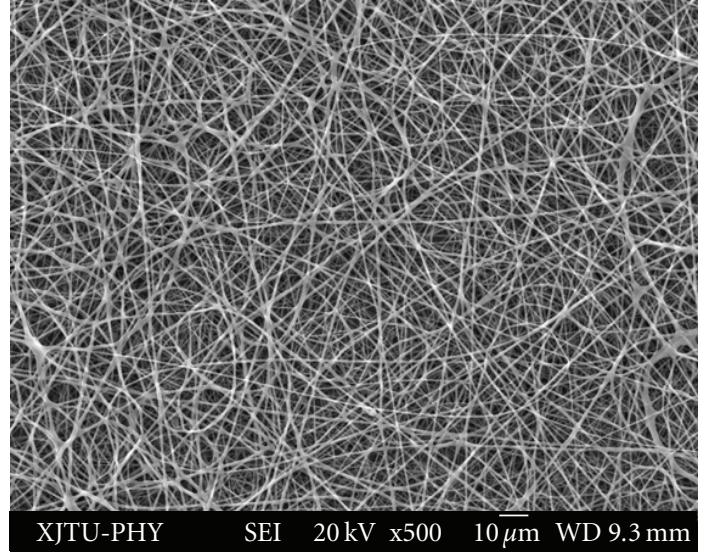

(a)

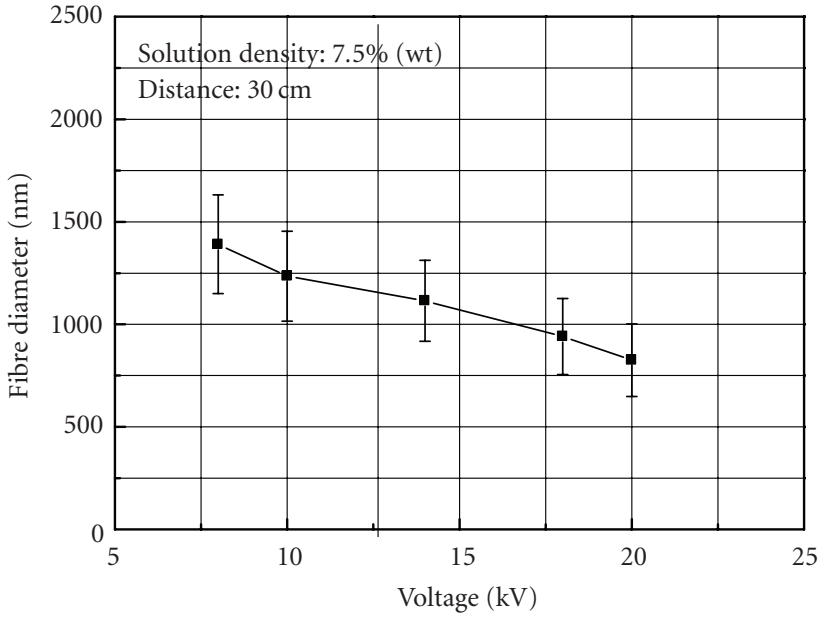

(c)

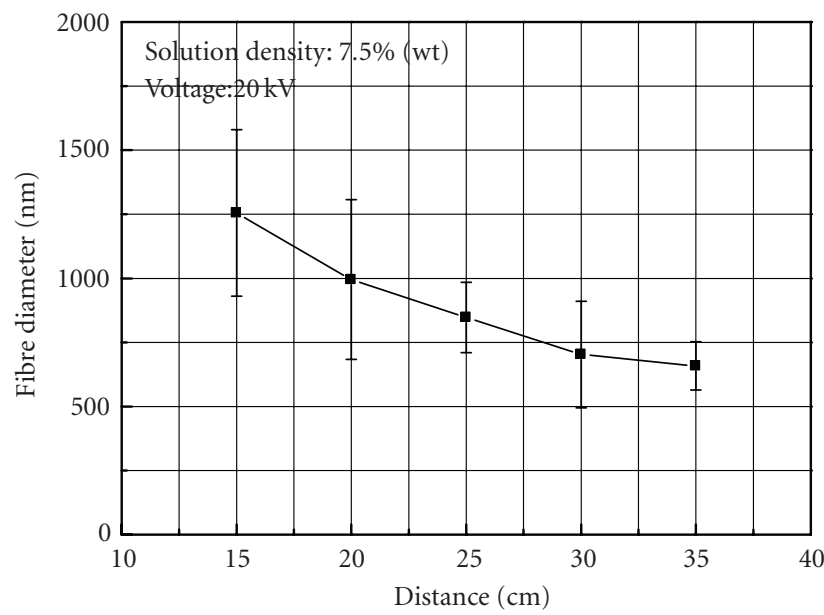

(e)

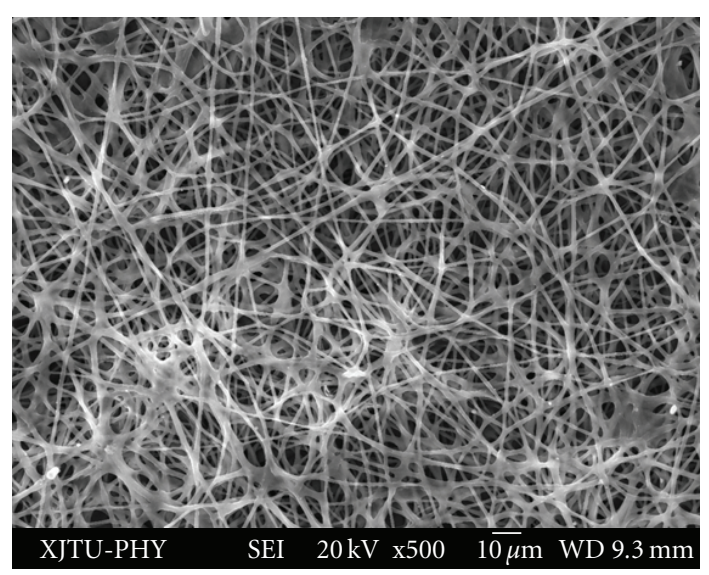

(b)

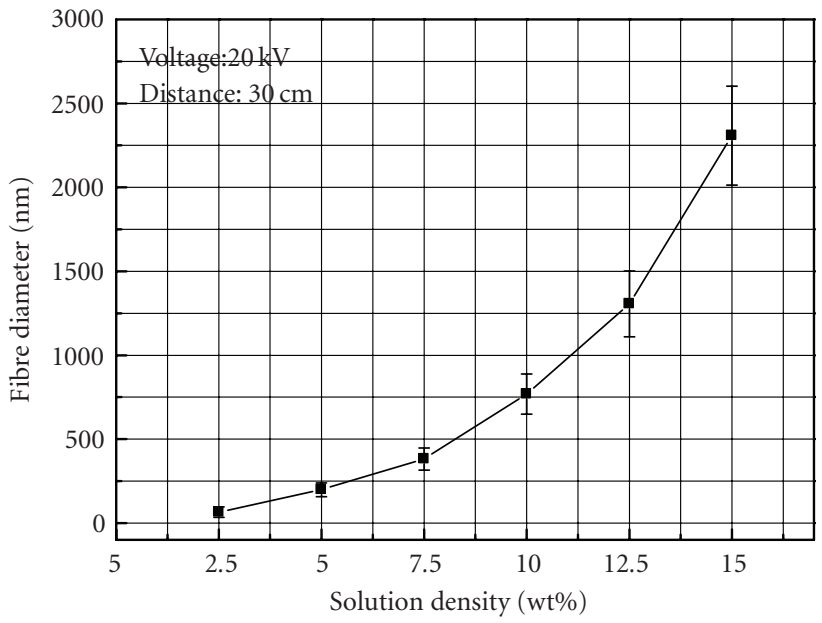

(d)

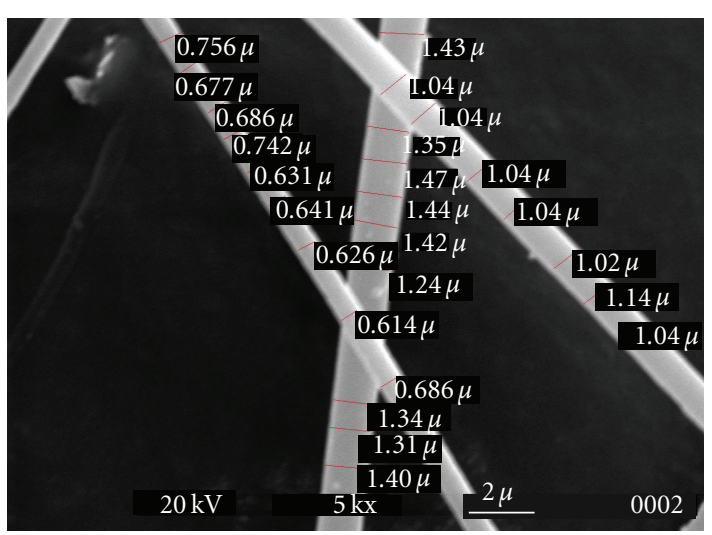

(f)

FIGURE 2: Nanofibres and fabrication parameters. (a) An SEM picture of EVOH nanofibres produced with a solution in a stabilized temperature; (b) an SEM picture of EVOH nanofibres with a solution in decreasing temperature; (c) effect of the voltage on fibre diameters when the solution density is $7.5 \%$ (wt) and needle tip-to-collector distance is $30 \mathrm{~cm}$; (d) effect of the density on fibre diameters when the voltage is $20 \mathrm{kV}$ and the tip-to-collector distance is $30 \mathrm{~cm}$; (e) effect of the distance on fibre diameters when the solution density is $7.5 \%$ (wt) and the voltage is $20 \mathrm{kV}$; (f) an example of SEM measurement of fibre diameters. 
conductive tapes $\left(3 \times 3 \mathrm{~mm}^{2}\right)$ to make 12 fibre samples from each experiment directly in different areas. All diameters of the 12 samples were measured under SEM (Figure 2(f)) long each fibre. Due to the variation of fibre diameters, around 1000 measurement were carried out in total for statistic evaluation. Figures 2(c)-2(e) show the average values. Based on the results, calculations were carried out for the 3 main control parameters, that is, the tip-to-collector distance, the field voltage, and the solution density.

In addition, another important parameter which controls the fibre formation is the injection rate of the $\mathrm{EVOH}$ solution. This is normally done through the feeding speed control of the micropump. However, our study shows that there is only a very narrow window of the speed which can be utilized to produce fibres. In other words, there is no much room to play with the injection speed. One either gets it right for clear fibres, or wrong for no fibres or poor fibre formations. Thus, the injection speed is not really a "controllable" parameter. In this study, the speed we used was $1.5 \mathrm{ml} / \mathrm{h}$ which allows us to obtain fibres in the diameter range from 100 to 1000 nanometers.

2.6. Bacteria Test. Bacteria test was performed to test the ability of restraining pathogens using the fabricated Agencapsulating nanofibres. Staphylococcus aureus, which is one of the main pathogenic bacterias found on animal/human burn surface, was used in this study. Staphylococcus aureus was diluted by LB culture fluid to OD600 value 1.1. $0.5 \mathrm{~mL}$ of the diluted fluid was used to fully cover the base of a culture dish. Four fibre samples with different concentrations of $\mathrm{Ag}$ nanoparticles were tiled into the culture dishes. The culture was then carried out in an incubator at shaking speed of $50 \mathrm{r} / \mathrm{min}$ for 6 hours at $37^{\circ} \mathrm{C}$. To quantify the effectiveness of restraining pathogens, we measured the bacteriostatic loops at time intervals of 6 hours using a digital camera, and the diameters were measured by an electronic venire caliper.

\section{Results and Discussions}

3.1. Fabrication Parameters. EVOH is thermally sensitive which may affect the electrospinning process. To check the effect of temperature, we used SEM to assess the morphology of the nanofibres fabricated with/without temperature control during the fabrication. The temperature of the solution was controlled either at $80^{\circ} \mathrm{C}$ constantly or allowed to cool down from $80^{\circ} \mathrm{C}$ to room temperature naturally during the spinning process, respectively. Comparison of Figures 2(a)-2(b) shows that the quality of fibres deteriorates when solution temperature is decreasing (Figure 2(b)), that is, fibres become sticky to form clusters with larger and uneven diameters. Figure 2(a) also shows larger interfibre space which is beneficiary for moisture regulation [19].

It is reported that the fibre diameter may influence the healing speed [20]. To evaluate the control of the fibre diameter, we quantified the relationship between the fibre diameter and the 3 parameters discussed, that is, the tipto-collector distance, the voltage of the electric field, and the density of polymer solution. The findings are illustrated in Figures 2(c)-2(e). Figure 2(c) shows the effect of voltage for the cases with a constant solution density at $7.5 \%$ and the tip-to-collector distance at $30 \mathrm{~cm}$. We observed that the most suitable voltage range is from 10 to $20 \mathrm{kV}$. At lower voltages $(<10 \mathrm{kV})$, there is no enough power to drive the $\mathrm{EVOH}$ jet through the electric filed to form nanofibres, while at a higher value $(>20 \mathrm{kV})$, the solution jet is broken by the too strong stretching force by the electrical field that no continuing fibres can be formed. Figure 2(d) shows results of 6 different values of solution density at 2.5, 5.0, 7.5, 10, 12.5 , and $15.0 \mathrm{wt} \%$, respectively. The voltage was kept at $20 \mathrm{kV}$ and the tip-to-collector distance at $30 \mathrm{~cm}$. It is clearly shown that the fibre diameter increases rapidly in increasing solution densities. The increased density leads to a higher viscosity which makes it more difficult to stretch the jet into finer fibres and also more prone to form "blockage" at the needle tip. We also noted that the fibre diameter can be influenced by the environmental temperature and humidity level. Higher temperature and low humidity are found to lead to finer fibre formation, though we were not able to quantify this. An effort to increase the temperature in the spinning space led to air circulation and yielded severe disturbance to fibre collection. Thus, all our results were obtained in ambient environmental conditions. Figure 2(e) shows results of 5 tip-to-collector distances of 15, 20,25, 30 and $35 \mathrm{~cm}$, respectively. $20 \mathrm{kV}$ field voltage and $7.5 \mathrm{wt} \%$ solution density were used. We observed no significant effect of the distance on the fibre diameter. Nevertheless, the distance provided a space necessary for fibres to stretch and dry up before reaching the collector. Otherwise, fibres will stick together to form clusters. In our tests, this occurs when the distance was reduced to $10 \mathrm{~cm}$. On the other hand, when the distance was increased to $40 \mathrm{~cm}$, the fibres were not collectable due to too violent spinning.

3.2. Energy Spectrum Check of Encapsulated Ag Particles. The suppressive effect of $\mathrm{Ag}$ on inflammation is related to the purity Ag (simple substance) encapsulated. To check the content of the Ag particles obtained, the energy spectrum function of the SEM was used. Figures 3(e)-3(f) show the composition of the $\mathrm{Ag}$ particles and the image of the $\mathrm{Ag}$ particle in the fibres. It shows that the particles are simple substance $\mathrm{Ag}$, where the elements $\mathrm{C}$ and $\mathrm{O}$ are from the EVOH polymer.

3.3. Bacteria Culture Tests. The main pathogens on the surface of skin burn is Staphylococcus aureus (>80\%) [21]. To assess the pathogen-restraining ability of the Agencapsulating nanofibres, we performed bacteria tests on EVOH fibres with different Ag concentrations (Figure 3(b)). We produced 4 samples of different Ag content, that is, 0.03 (Figure 3(b)-1), 0.07 (Figure 3(b)-2), 0.1 (Figure 3(b)3 ), and 0.15 (Figure 3(b)-4) g, respectively, in $10 \mathrm{~mL} 7.5$ wt $\%$ 80\%/20\% 2-propanol/water solution, respectively, and EVOH fibres without Ag (Figure 3(g)). Results of the bacteria tests are based on the measurements of bacteriostatic loop. The diameter of their bacteriostatic loops [22] was measured after culture (Figure 3(c)). A linearly increasing effectiveness 


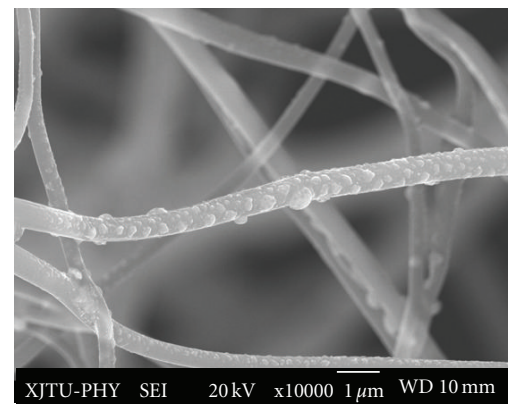

(a)

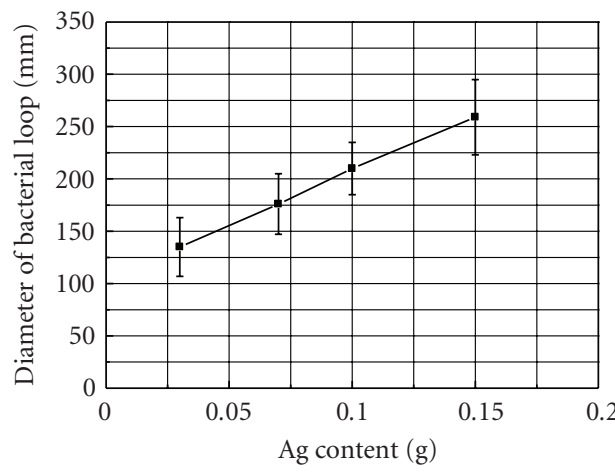

(c)

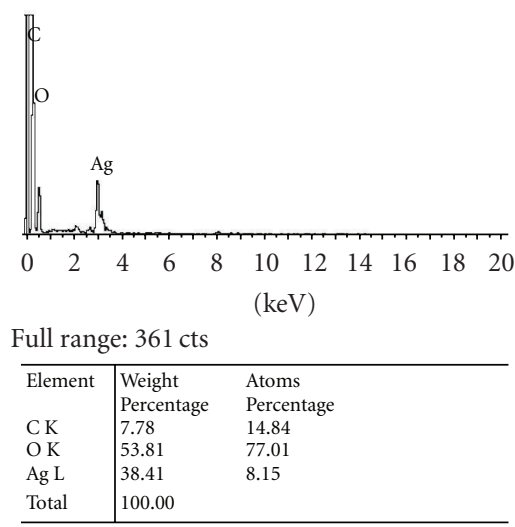

(e)

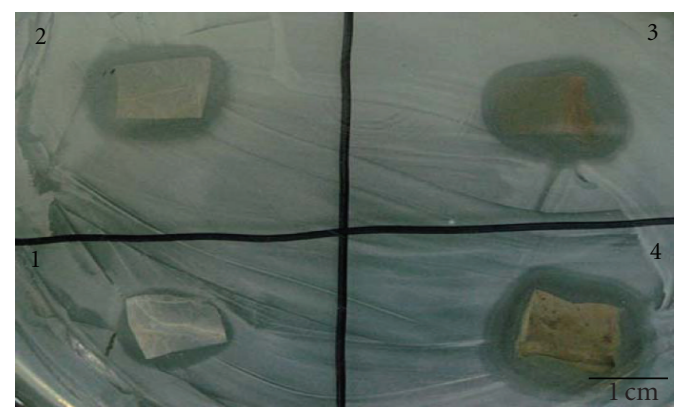

(b)

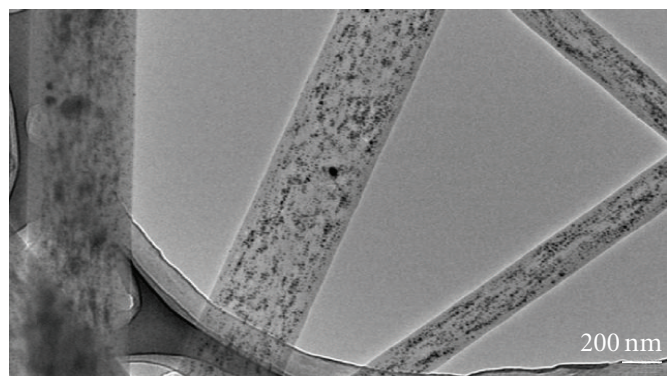

(d)

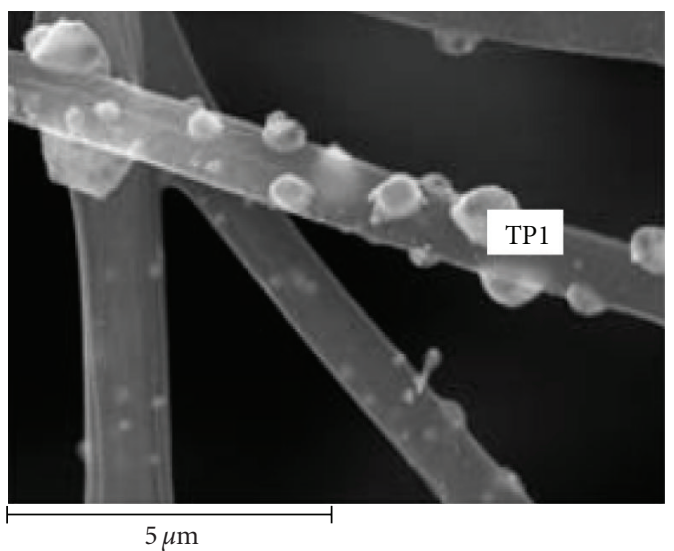

(f)

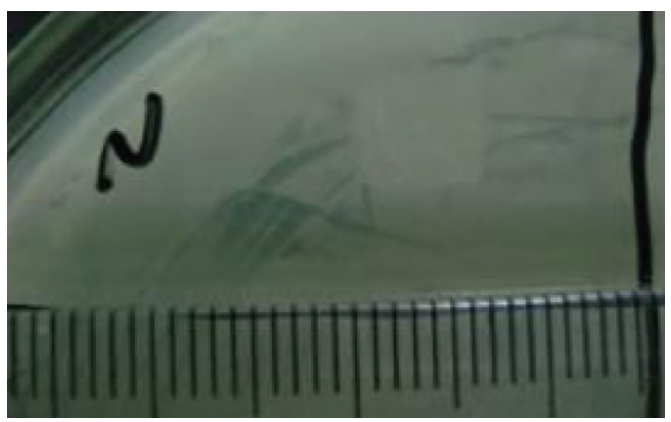

(g)

FIGURE 3: Fabrication Ag-encapsulated nanofibres and bacteria tests. (a) SEM image of nanofibre encapsulated with Ag nanoparticle (Tipto-collector distance is $30 \mathrm{~cm}$, voltage is $20 \mathrm{kV}$, and solution density is $7.5 \mathrm{wt} \%$ ), (b) image of one set of culture test for bacteriostatic loop measurement, (c) averaged measurement of bacterial loop diameter versus Ag concentration (wt/10 mL), (d) a TEM image encapsulated with Ag nanoparticle (Tip-to-collector distance is $30 \mathrm{~cm}$, voltage is $20 \mathrm{kV}$, and solution density is $7.5 \mathrm{wt} \%$ ), (e) and (f) energy spectrum using SEM for encapsulated Ag particles, and (g) image of EVOH fibre without Ag particles in the bacteria test. 
is found amongst the four concentrations used, with the highest Ag concentration yielding in the biggest loop for the same area of the fibre patches, clearly indicating a stronger pathogen-restraining effect. In contrast, no bacteriostatic loop was observed for pure EVOH fibres with Ag, indicating no pathogen restraining ability for fibres without Ag (Figure 3(g)).

In this study, no biodegradable tests were carried out on the nanofibres though this feature of the EVOH is well known [17]. The challenges remain in the simulation of the temperature, moisture, and importantly the $\mathrm{PH}$ value in a skin wound environment and their variations during the application of the fibres. However, this is a potentially important feature as it may determine the release speed of the Ag particles not on fibre surface and drugs which can be contained in the fibre. This remains a key test to be conducted in the following study.

\section{Conclusions}

Electrospinning is a straightforward approach to fabricate highly fibrous and porous EVOH materials for medical applications. In this study, we fabricated EVOH nanofibre encapsulated with Ag nanoparticles. The results of fibre characterization show that the nanofibre size can be controlled by regulating $\mathrm{EVOH}$ solution concentration, voltage, and distance of the electric field. Material characterization shows that $99 \%$ pure Ag particles can be formed in the fibre with $\mathrm{AgNO}_{3}$ added to the solution. Adding high concentration of Ag might change the fibre morphology; however, only low concentrations of Ag were used in our experiments. Compared under SEM and TEM observations, the morphology of Ag particles fibres is of no significant difference with pure EVOH fibres. Results of bacteria tests show that pathogen-restraining ability of the Ag-encapsulated nanofibres is effective and proportional over a range of $\mathrm{Ag}$ concentration, indicating its inflammation control capacity and the potential for applications in skin wound treatment. To further evaluate the suitability and effectiveness of Agencapsulating nanofibres on skin wound healing, animal tests have been planned in the next stage of study.

\section{Acknowledgment}

The work was partially supported by the National Natural Science Foundation of China (nos. 10825210, 10872157, and 31050110125) and the National 111 Project of China (no. B06024).

\section{References}

[1] F. Xu, P. F. Wang, M. Lin, T. J. Lu, and E. Y. K. Ng, "Quantification and the underlying mechanism of skin thermal damage: a review," Journal of Mechanics in Medicine and Biology, vol. 10, no. 3, pp. 373-400, 2010.

[2] L. Zhou, Y. Hu, and M. Zhang, "Investigation on the psychological conditions of face or body burned patients," Journal of North China Coal Medical College, vol. 10, no. 4, pp. 503-504, 2008 (Chinese).
[3] M. Choinière, R. Melzack, N. Girard, J. Rondeau, and M. J. Paquin, “Comparisons between patients' and nurses' assessment of pain and medication efficacy in severe burn injuries," Pain, vol. 40, no. 2, pp. 143-152, 1990.

[4] P. A. Brigham and E. McLoughlin, "Burn incidence and medical care use in the United States: estimates, trends, and data sources," Journal of Burn Care and Rehabilitation, vol. 17, no. 2, pp. 95-107, 1996.

[5] R. L. Sheridan, "Burn care: results of technical and organizational progress," Journal of the American Medical Association, vol. 290, no. 6, pp. 719-722, 2003.

[6] B. H. Yu and J. E. Dimsdale, "Posttraumatic stress disorder in patients with burn injuries," Journal of Burn Care and Rehabilitation, vol. 20, no. 5, pp. 426-433, 1999.

[7] I. Calif, "U.S. markets for wound management products," Medical Data International, 1997.

[8] F. Xu, T. J. Lu, K. A. Seffen, and E. Y. K. Ng, "Mathematical modeling of skin bioheat transfer," Applied Mechanics Reviews, vol. 62, no. 5, Article ID 050801, 35 pages, 2009.

[9] F. Xu and T. J. Lu, "Skin biothermomechanics: modeling and experimental characterization," in Advances in Applied Mechanics, E. van der Giessen and H. Aref, Eds., pp. 147-248, Academic Press, Burlington, Mass, USA, 2009.

[10] B. Domres, D. Kistler, and J. Rutczynska, "Intermingled skin grafting: a valid transplantation method at low cost," Annals of Burns and Fire Disasters, vol. 20, no. 3, pp. 149-154, 2007.

[11] C. R. Carlisle, C. Coulais, M. Namboothiry, D. L. Carroll, R. R. Hantgan, and M. Guthold, "The mechanical properties of individual, electrospun fibrinogen fibers," Biomaterials, vol. 30, no. 6, pp. 1205-1213, 2009.

[12] L. E. Claes, "Mechanical characterization of biodegradable implants," Clinical Materials, vol. 10, no. 1-2, pp. 41-46, 1992.

[13] A. A. Ignatius and L. E. Claes, "In vitro biocompatibility of bioresorbable polymers: poly(L, DL-lactide) and poly(Llactide-co-glycolide)," Biomaterials, vol. 17, no. 8, pp. 831839, 1996.

[14] J. F. Cooley, "The process of electro-spinning," US patent, 1902.

[15] G. Taylor, "Electrically driven jets," Proceedings of the Royal Society of London. Series A, vol. 313, no. 1515, pp. 453-475, 1969.

[16] Y. Dzenis, "Spinning continuous fibers for nanotechnology," Science, vol. 304, no. 5679, pp. 1917-1919, 2004.

[17] E. Kenawya, J. M. Layman, J. R. Watkins et al., "Electrospinning of poly(ethylene-co-vinyl alcohol) fibers," Biomaterials, vol. 24, no. 6, pp. 907-913, 2003.

[18] P. Zhang, S. Phansiri, and J. Puonti-Kaerlas, "Improvement of cassava shoot organogenesis by the use of silver nitrate in vitro," Plant Cell, Tissue and Organ Culture, vol. 67, no. 1, pp. 47-54, 2001.

[19] M. Zhang, Y. S. Huang, and Q. Zhang, "Protective effect of nitric oxide production on myocardium in severely scalded rats," Chinese Journal of Burns, vol. 23, no. 5, pp. 327-330, 2007.

[20] C. B. D. J. Hinsey, "The recovery of diameter and impulse conduction in regenerating nerve fibers," Annals of the New York Academy of Sciences, vol. 47, no. 4, pp. 559-574, 1946.

[21] A. S. Colsky, R. S. Kirsner, and F. A. Kerdel, "Analysis of antibiotic susceptibilities of skin wound flora in hospitalized dermatology patients: the crisis of antibiotic resistance has 
come to the surface," Archives of Dermatology, vol. 134, no. 8, pp. 1006-1009, 1998.

[22] Q. Zheng, H. Wu, J. Du, S. Li, and Y. Yan, "The bacterial inhibitory ability and in vivo drug release pattern of a new drug delivery system: ciprofloxacine/tricalcium phosphate delivery capsule," Journal of Huazhong University of Science and Technology. Medical Sciences, vol. 18, no. 3, p. 172176, 1998. 

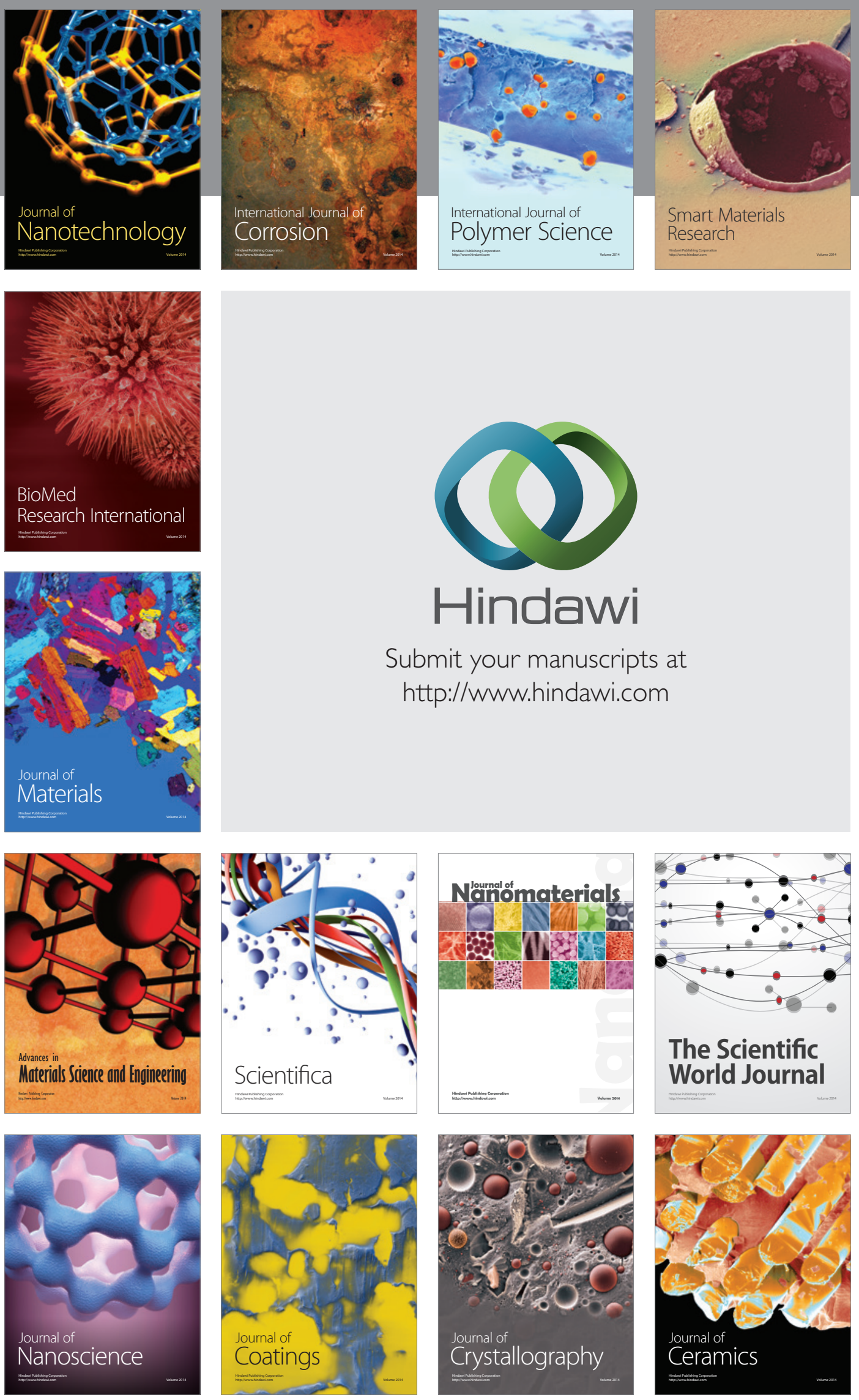

The Scientific World Journal

Submit your manuscripts at

http://www.hindawi.com

\section{World Journal}

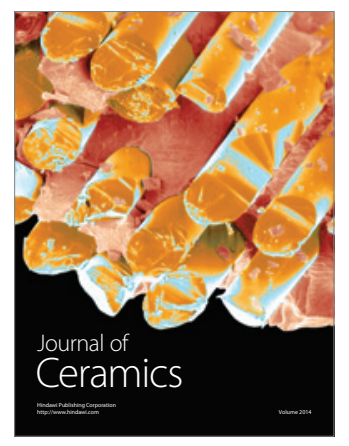

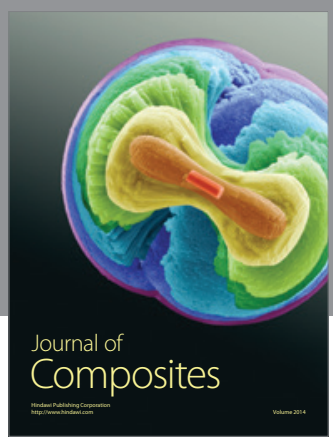
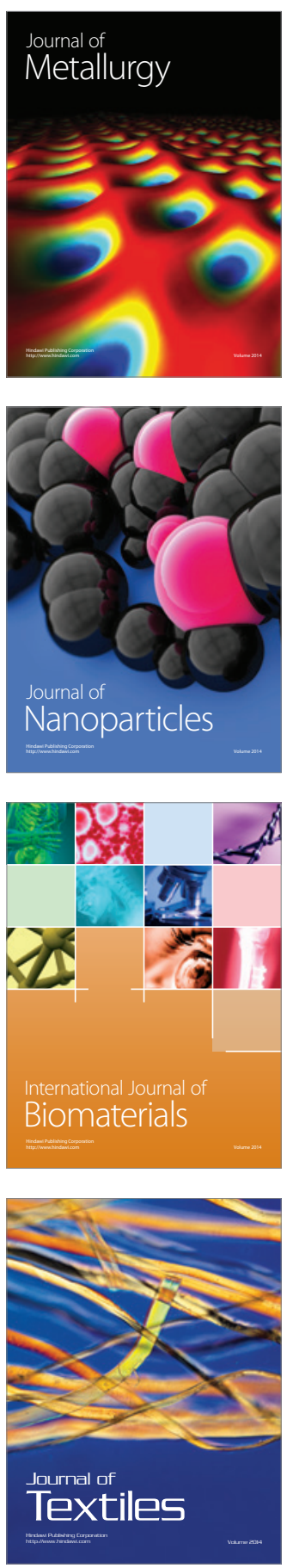